\title{
Integration of a RSI microstructure sensing package into a Seaglider
}

\author{
Elizabeth Creed and William Ross \\ Kongsberg Underwater Technology, Inc. \\ Lynnwood, WA, USA
}

\author{
Dr. Rolf Lueck, Peter Stern, William Douglas and \\ Dr. Fabian Wolk \\ Rockland Scientific, Inc. \\ Victoria, BC, Canada
}

\author{
Dr. Rob Hall \\ Centre for Ocean and Atmospheric Sciences \\ School of Environmental Sciences \\ University of East Anglia \\ Norwich, UK
}

\begin{abstract}
Seagliders are a type of propeller-less AUV that glide through the water by changing their buoyancy. They have become mainstream collectors of standard oceanographic data (conductivity, temperature, pressure, dissolved oxygen, fluorescence and backscatter) and are increasingly used as trucks to carry a wide variety of hydrographic and bio-geochemical sensors. The extended sensor capability enhances the utility of the gliders for oceanographic observations.

Seagliders are designed and optimized for long-term missions (up to 10 months) and deep sea profiling (up to $1000 \mathrm{~m}$ ). They provide high resolution oceanographic data with very good temporal and spatial density, in near real-time, at a fraction of the cost of ship collected data. These performance parameters are sometimes at odds with the physical dimensions and electrical requirements of the hydrographic and bio-geochemical sensors scientists want installed in gliders. However, as the acceptance of gliders as an integral component of the oceanographic suite of measurement tools grows so do the efforts of sensor vendors to develop products that meet the size, weight and power requirements for successful glider integration.

Turbulence microstructure sensors are one measurement system that scientists desired on Seagliders but that until recently did not fit the glider footprint. In collaboration with Rockland Scientific, Inc., a suite of RSI turbulence microstructure sensors was recently integrated into a Seaglider and the system's performance validated during field tests in Puget Sound near Seattle, WA and in Loch Linnhe on the west coast of Scotland. Ocean turbulence controls the mixing of water masses, biogeochemical fluxes within them, and facilitates oceanatmosphere gas exchange. As a result, turbulence impacts global ocean circulation, polar ice melt rates, drawdown of atmospheric carbon dioxide and carbon deposition, coastal and deep ocean ecology, commercial fisheries, and the dispersion of pollutants. Turbulent mixing is also recognized as a key parameter in global climate models, used for understanding and predicting future climate change. Seagliders equipped with turbulence microstructure sensors will allow scientists to map the geographical distribution and temporal variability of mixing in the ocean on scales not possible with ship-based measurements.
\end{abstract}

This presentation discusses the technical aspects of the integration of the turbulence sensor suite on a Seaglider with an emphasis on achieving high data quality, while retaining the performance characteristics of the Seaglider. We will also describe applications for this sensor suite, examine the turbulence measurement data already collected by the Seaglider and discuss future deployment plans.

Keywords- sensor integration, MicroPod, Seaglider, microstructure measurements

\section{INTRODUCTION}

Early underwater gliders were designed to carry a limited science sensor payload that had a small size, weight and power consumption footprint. The ideal early glider system travelled at 0.5 knots using 0.5 Watts of power. However, as the popularity of gliders has grown so has the desire for them to be able to carry larger science payload packages while maintaining the efficiency needed to remain deployed for multiple month missions. The Rockland Scientific,4 Inc. (RSI) microstructure sensing system is an example of a science payload package that was reconfigured from its original design for use specifically on a Seaglider.

\section{INTEGRATION TECHNICAL CHALLENGES}

Incorporating RSI's microstructure technology onto the Seaglider presents challenges. The Seaglider's body profile and fairing smoothness were optimized for drag and lift. The addition of a sensor package that must measure in undisturbed water while minimizing its effect on the glider's flight characteristics requires a new approach to RSI's traditional instrumentation. On other platforms, RSI has provided an "allin-one" payload where the sensors and supporting electronics are contained within a single housing that only requires power and an ON/OFF signal from the supporting platform. These self-contained units (for example, the MicroRider) are mounted 
onto the supporting platform such that the sensors are above and forward of the bow of the platform where flow distortion is small. The MicroRider cannot be used on the Seaglider because of a geometry mismatch that makes its mounting difficult, and the size of the MicroRider adds an unacceptable drag. The Seaglider also requires a more sophisticated software interface so that the RSI system is fully controlled by the standard Seaglider scientific logger payload control software, Logdev.

\section{A. Design Modifications}

The reconfigured payload package consists of two MicroPod units mounted to the Seaglider's aft fairing, one on either side of the CT sail, and a dedicated pressure housing, containing the system's DataLogger, mounted inside the glider's flooded aft fairing, Fig. 1. This distributed system allows the direct exposure of the sensing surfaces to 'clean water' while preserving the glider's low drag fairing profile.

The MicroPod housings are machined from Acetal plastic into a cylindrical shape with a tapered nose for hydrodynamic efficiency. Depth rated to $1000 \mathrm{~m}$, the housing diameter is 3.8 $\mathrm{cm}$ (1.5 in) with an overall length of $27.9 \mathrm{~cm}$ (11 in), including the probe. Impulse IE55 connectors are used to match the standard connectors on the Seaglider. The MicroPods are designed to handle either a shear probe or a FP07 thermistor. The electronic circuit board located inside the MicroPod is specific to either the shear probe or the thermistor and can be changed as needed, Fig. 2.

The probes for the MicroPod are slightly different from standard RSI probes. Standard RSI sensors use a threaded SMC type coaxial connector, whereas the sensors for a MicroPod use a SMB plug-in type connector. The cables
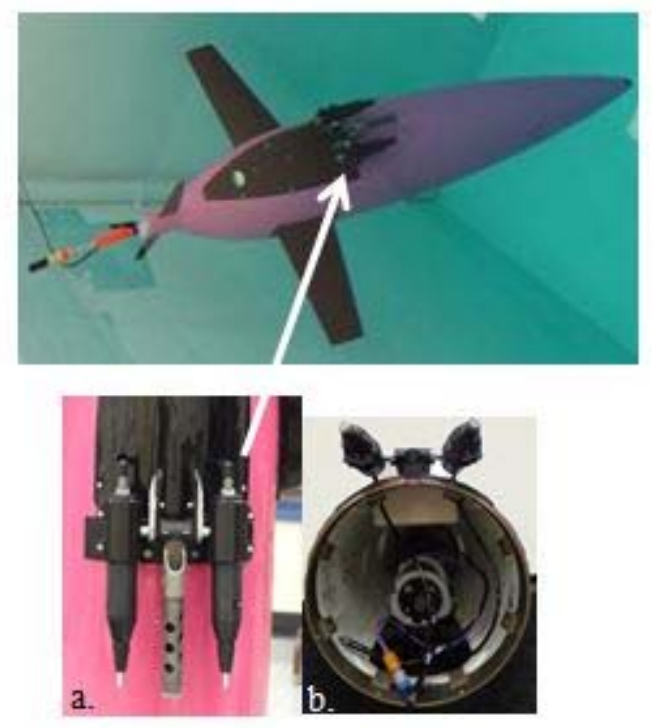

Fig. 1. Seaglider with microstructure sensing package. Inset a. is an enlargement of the MicroPod assembly. Inset b. shows the placement of the microstructure DataLogger pressure case inside the aft fairing of the glider in relation to the MicroPods.

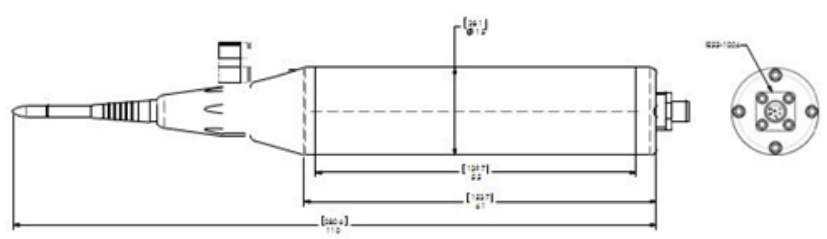

Fig. 2. Drawing of a MicroPod with probe installed.

connecting the MicroPods to the DataLogger are routed via a right-angle connector vertically through the hull. The passthrough points are located in the upper hatch cover directly above the DataLogger pressure case to minimize the cable length. The shear probe is directionally sensitive and can be rotated around its longitudinal axis to measure any athwartship component of velocity. The direction of sensitivity of the shear probe, and the vibration sensor, are easily changed from nominally vertical to lateral by removing the single screw that retains the front bulkhead, rotating the bulkhead ninety degrees, and re-securing the screw. The screw is the alignment mark. The FP07 housing is identical to the shear housing, but it has no vibration sensor, and the FP07 is not direction sensitive.

The MicroPods are mounted to the aft fairing of the Seaglider using a Delrin and 316 stainless steel bracket designed by Kongsberg. The mounting bracket can be rotated from parallel to the major (longitudinal) axis of the glider to ten degrees above the axis. This allows tests to be carried out on: (a) effect of angle between the shear probe and the direction of glider travel on the measured shear spectrum, and (b) effect of distance between the probes and the glider body on estimated dissipation rates.

In the present configuration a shear probe, oriented to sense the nearly vertical (athwartship) fluctuations of velocity, and a piezo accelerometer to sense vibrations in the same direction are installed in one MicroPod unit. The electronics produce analog voltages that are proportional to the rate of change of velocity and acceleration. Both signals are high-pass filtered at $0.1 \mathrm{~Hz}$. The second MicroPod holds a FP07 thermistor to sense temperature and its fluctuation. The thermistor electronics produce an analog voltage that is nearly linear with respect to temperature plus its rate of change.

The DataLogger pressure case is made of anodized 6061T6 aluminum and rated to $1500 \mathrm{~m}$ with a safety factor of 1.47 by Finite Element Analysis (FEA). Its outer dimensions are $30.5 \mathrm{~cm}$ (12 in) long (including the bulkhead connectors) by $8.9 \mathrm{~cm}$ (3.5 in) diameter. It is secured inside the aft fairing of the Seaglider by two Delrin clamps that are screwed to the lower hatch cover. The single endcap is sealed by two O-rings. There are four IE55 bulkhead connectors located on the endcap, one for each MicroPod, one to connect the DataLogger pressure case to the Seaglider's aft endcap and the fourth to connect a USB jumper cable that runs to the glider's rudder shoe and allows direct communication to the pressure case without the need for direct access to it. A sacrificial anode is also located on the endcap.

The DataLogger has four components; a Power Supply board, a General Purpose Analog Input board, a CF2 interface 
with a Persistor CF2 computer and a newly developed Platform Interface board. The Power Supply board uses input from the glider during normal operations and from an external source during direct communications operations such as data download after a mission.

The Platform Interface board performs a number of functions. It allows the Seaglider to control the microstructure sensor via a RS-232 communications link; e.g. starting and stopping data acquisition and initiating the collection and transfer of snippet files to the Seaglider shore-side command computer during the bi-directional file exchange that occurs between the glider and the command computer at each surfacing. To accomplish this, the Platform Interface board responds to Seaglider requests to power on and off by applying the required signal to the DataLogger. The Platform Interface board then waits for verification and returns a status message to the Seaglider. This allows the Seaglider to turn DataLogger power on and off without the danger of file system corruption. The Platform Interface board also performs the job of associating glider dive file names requested by Kongsberg with data files generated by the microstructure DataLogger. This allows Kongsberg's Seaglider to download snippet files using existing protocols thereby not requiring major changes to the glider software. Finally, the Platform Interface board permits a more streamlined connection by the user on the deck to access the RSI system. A USB hub is embedded into the Platform Interface board to facilitate both the USB virtual serial port, which allows console access to the DataLogger, and USB data transfers over the single physical USB connection on the deck cable. When the externally powered RSI deck cable is plugged into the DataLogger it triggers the RSI system to turn on and initiate the USB data transfer mode. Operators can then use the RSILink application to download data files from the DataLogger without first explicitly initiating the USBL.RUN via console interaction.

The MicroPod signals are sampled at a rate of $512 \mathrm{~s}^{-1}$ and these records, along with the pitch and roll of the DataLogger pressure case and the voltage of the power supplied by the Seaglider, are stored on the Compact Flash card installed on the Persistor CF2 computer. Rockland Scientific's standard data logging software, ODAS-IR (Ocean Data Acquisition System - Internally Recording), is installed in the flash memory of the Persistor CF2 computer and manages the data logging functions. ODAS-IR uses a separate setup file on the Compact Flash card to set the data logging parameters. This text file is edited by the user prior to deployment to reflect the specific microstructure sensors installed in the MicroPods. The setup file directs the logging sequence and the sampling rates for each channel.

The turbulence system does not record depth or speed. This information, which is crucial to converting the data into physical units and its scientific interpretation, is derived from the data files collected by the Seaglider during a dive and processed by the command computer into a net-CDF file. The clock in the microstructure sensor electronics and the clock in the Seaglider are synchronized to GPS at every glider surfacing allowing for easy interpolation of the glider data into the turbulence data. The concurrent records of pitch and roll provide confirmation of the time synchronization of these two independent instrument systems.

\section{B. Technical Considerations}

The mechanical design of the MicroPods and the DataLogger pressure housing went smoothly. The MicroPods were assembled and tested without problems. Initial testing of the MicroPod mounting on the Seaglider showed that the fixture was excessively flexible and may have vibrated enough to obscure real oceanic velocity signals. Minor modifications were made to the mounting bracket to reduce lateral and vertical motion and prevent the mounting angle adjustment mechanism from slipping.

The DataLogger pressure case could not be mounted in the preferred position with its longitudinal axis parallel to the major axis of the glider due to the ogive fairing shape and space constraints within the fairing. This means that the pitch angle measured by a precision $\left( \pm 0.1^{\circ}\right)$ inclinometer on the Power Supply board is offset from the angle measured by the glider's navigation system. It is also not possible to rotate the pressure case so that the roll measured by the inclinometers coincides with the roll measured by the glider's navigation system, because of interference of the DataLogger pressure case bulkhead connectors with the glider's buoyancy bladder. An offset has been applied to address the pitch issue. Orientation of the boards inside the pressure case will be changed in the next version of the design so that the roll measured by the inclinometers coincides with the roll measured by the glider's navigation system.

Some challenges occurred in the commissioning of the newly developed Platform Interface board. The most significant challenge was providing a robust mechanism for installing the USB drivers required to download the raw data files from the DataLogger after the completion of a glider mission. Running RSILink on both Windows and MacOS proved difficult, but each for a different reason. On Windows it was hard to get device drivers to install correctly. On MacOS there was a conflict between the driver provided within the latest version of the operating system and the driver embedded within the application. The issue was remedied by using a custom USB identifier to uniquely identify the CF2 board and modifying RSILink to use the new identifier.

\section{PRELIMINARY TESTING}

Eliminating noise from the shear probe signal is usually the biggest obstacle to using this sensor. The noise is almost always from mechanical vibrations of the platform holding the probes, but unwanted signals can also be induced by electric fields and acoustic transducers. For laboratory work, the shear probes must be well shielded from $60 / 50 \mathrm{~Hz}$ electromagnetic fields. Similar shielding is required for the piezoaccelerometers. The common practice of using aluminum foil to shield the probes was applied during the bench testing. In natural waters this form of radiation is extremely small.

The first half day deployment of the system in Shilshole Bay, Puget Sound, WA revealed a pernicious source of noise. In this test the shear probe, vibration sensor and the battery voltage displayed significant spiking in intervals of $5 \mathrm{~s}$. This 
contamination was absent from the MicroPod thermistor signal. Intentional timing variations of the activation of the buoyancy engine, and the sampling of the altimeter and CT sensor did not reveal the source of the spikes. A conscious decision was made at the start of the integration to not capacitively ground the microstructure sensor to seawater due to concerns over corrosion, interaction with electric systems internal to the Seaglider, and wireless telemetry. However, after this noise was observed it was decided to follow RSI's standard practice of grounding their instrumentation to seawater via a single capacitor. A subsequent half day deployment demonstrated that the capacitive grounding of the DataLogger removed the spikes in the shear probe signal.

\section{ENGINEERING SEA TRIAL}

As a final engineering test of the system, a Seaglider carrying the integrated microstructure measurement system was deployed in Shilshole Bay, Puget Sound, WA, for 24 hours. Fifty-five turbulence profiles were collected. The FP07 thermistor was not calibrated prior to use so measurements made by it were converted into physical units using nominal coefficients. While the absolute value is off by as much as \pm 8 ${ }^{\circ} \mathrm{C}$, the trends are fairly accurate. The FP07 thermistor can be calibrated by comparing it to the thermistor on the glider.

The shear probe and thermistor signals show the typical depth-intermittency of turbulence, Fig. 3. Gradients are expressed with respect to $\mathrm{x}$, which is directed along the path of the glider. The $\mathrm{x}$-direction is only approximately horizontal and it is also not exactly parallel to the major axis of the glider, because there must be a small $\left(\sim 3^{\circ}\right)$ angle of attack in order to produce the lift required for gliding.

Significant "patches" of turbulence, at depths of 45, 95 and $135 \mathrm{dbar}$, are revealed by the shear probe signal (blue). Below 25 dbar depth, the vertical gradient of temperature is positive (black). The vertical fluctuations of turbulent velocity induce co-located fluctuations of the along-path gradient of temperature (red) and short vertical scale inversions of the temperature (black). The signals are low-pass filtered at $15 \mathrm{~Hz}$ for display purposes only.

The rate of dissipation of turbulence kinetic energy, $\varepsilon$, is estimated from the shear data using

$$
\epsilon=\frac{15}{2} \nu \overline{\left(\frac{\partial w}{\partial x}\right)^{2}}=\frac{15}{2} \nu \int_{0}^{k_{0}} \psi(k) \mathrm{d} k
$$

where $v$ is the kinematic viscosity, $\psi$ is the spectrum of shear, $\mathrm{k}$ $=\mathrm{f} / \mathrm{U}$ is the wavenumber, $\mathrm{f}$ the frequency, $\mathrm{U}$ the speed of profiling, and $\mathrm{k}_{\mathrm{u}}$ is the upper limit of spectral integration. A judicious choice of $\mathrm{k}_{\mathrm{u}}$ can eliminate signal contamination from irremovable vibrations and electronic noise. The empirically derived Nasmyth spectrum is often used to provide a guide to the quality of a measured spectrum.

A spectrum of the shear and temperature gradient for the segment between 90 and 105 dbar depth, Fig. 4, shows that the rate of dissipation of kinetic energy by the turbulence, $\varepsilon$ is 4.3 $\times 10^{-8} \mathrm{~W} \mathrm{~kg}^{-1}$, a significant but not unusual level of turbulence for a tidally driven inland sea. The speed of profiling is $0.38 \mathrm{~m}$ $\mathrm{s}^{-1}$. The limit of spectral integration is $50 \mathrm{cpm}$ (blue triangle). The most significant vibration is the spectral peak at $130 \mathrm{cpm}$ $(50 \mathrm{~Hz})$ which is only reduced by a factor of 10 by the coherent-noise removal algorithm (thin- versus thick-blue lines). However, for typical glider speeds it is well beyond the band of interest. A stiffening of the mount for the MicroPod reduced this vibration considerably in the subsequent science sea trial. There is a minor vibration at $55 \mathrm{cpm}(21 \mathrm{~Hz})$, which is detectable by the difference between the thin and thick blue lines but whose origin has not been determined. The vibration is eliminated by the coherent-noise removal algorithm.

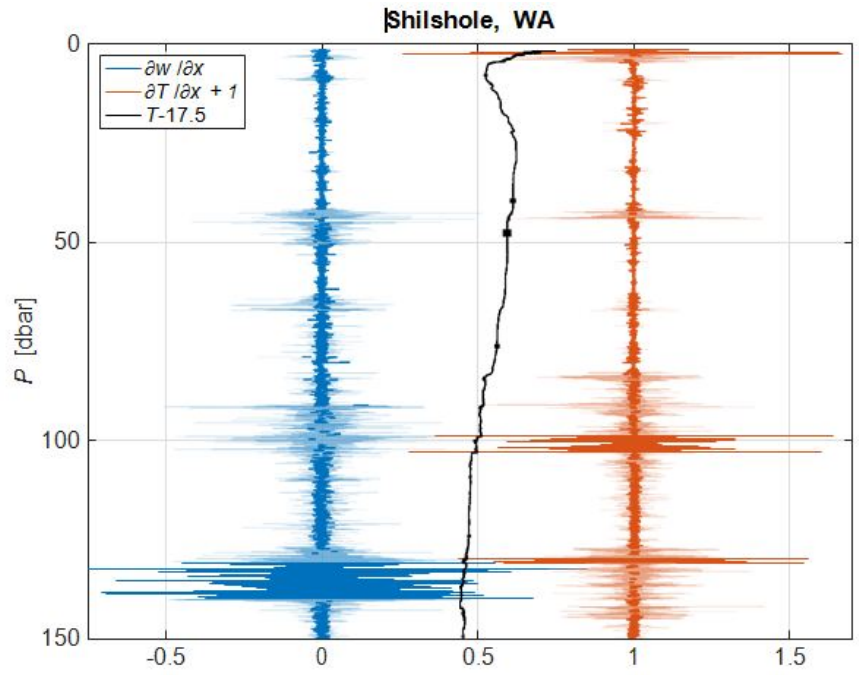

Fig. 3. Shear probe and thermistor signals showing the typical depthintermittency of turbulence; the along-path gradient of the fluctuation of the approximately vertical athwartship fluctuation of velocity (blue), the along-path gradient of temperature (red and offset by 1) and the temperature (black and offset by $17.5^{\circ} \mathrm{C}$ ) as a function of pressure, in units of dbar, which is nearly identical to depth in units of $\mathrm{m}$. The units are $\mathrm{s}^{-1},{ }^{0} \mathrm{C} \mathrm{m} \mathrm{m}^{-1}$ and ${ }^{0} \mathrm{C}$ for shear, temperature gradient, and temperature, respectively. The data are from the Puget Sound.

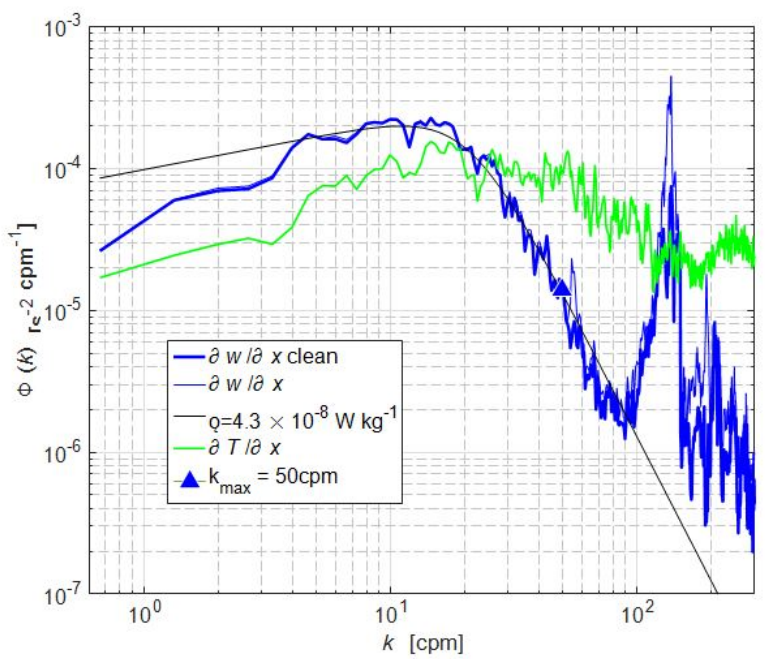

Fig. 4. The spectrum of vertical shear $(\partial w / \partial x$, thin blue line) from the Puget Sound in the pressure range of 90 to $105 \mathrm{dbar}$. The thick blue line is the spectrum of shear after removing contributions that are coherent with the vertical vibrations sensed by the piezo-accelerometer. The limit of spectral integration is $50 \mathrm{cpm}$ (blue triangle) and the black line is the Nasmyth spectrum for the estimated rate of dissipation. The temperature spectrum is in green. 
The shear spectrum agrees well with the Nasmyth spectrum (black) for wavenumbers between 4 and $100 \mathrm{cpm}$ but falls significantly below the Nasmyth spectrum at low wavenumbers. This is an artifact of orienting the shear probe to sense the vertical velocity fluctuations. The nearby hull of the glider suppresses this component of velocity fluctuations. That is, eddies with length scales small compared to the distance separating the shear probe from the hull will be unaffected by the hull, whereas those with larger scales will have a diminished velocity for the component that is normal to the hull.

There are very few data segments in which the turbulence is extremely low, and this makes it difficult to estimate the noise level of the instrumentation with statistical reliability. A segment centered on 38.6 dbar depth, spanning $1.4 \mathrm{~m}$ vertically, has a very low rate of dissipation, Fig. 5. The profiling speed is $0.41 \mathrm{~m} \mathrm{~s}^{-1}$. The major vibration at $130 \mathrm{cpm}$ and the one at $50 \mathrm{cpm}$ are similar to those shown in Fig. 4. The rate of dissipation, estimated by spectral integration to $10 \mathrm{cpm}$ is $2.6 \times 10^{-11} \mathrm{~W} \mathrm{~kg}^{-1}$ and the measured spectrum agrees reasonably well with the Nasmyth spectrum for wavenumbers smaller than $5 \mathrm{cpm}$. Vibration-coherent noise is evident at all wavenumbers but it is insignificant for wavenumbers smaller than $30 \mathrm{cpm}$.

\section{SCIENTIFIC SEA TRIAL}

The Seaglider and microstructure system were further tested in Loch Linnhe near Oban, Scotland between 22 and 26 June 2015. Four separate deployments were made, each lasting close to 24 hours. In total 85 dives were completed (74 deeper than 150 dbar) and 167 microstructure profiles were recorded. During three dives only a downward or upward profile was recorded to test the communication between the glider and microstructure system. In addition, microstructure data snippet

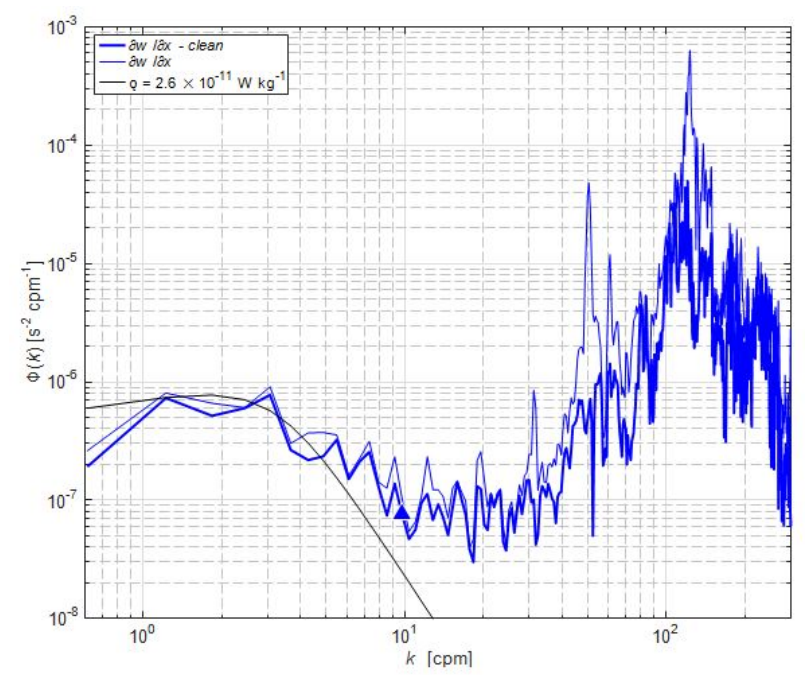

Fig. 5. The spectrum of vertical shear (thin blue line) from the Puget Sound in the pressure range of 37.9 to $39.3 \mathrm{dbar}$. The thick blue line is the spectrum of shear after removing contributions that are coherent with the vertical vibrations sensed by the piezo-accelerometer. The limit of spectral integration is $50 \mathrm{cpm}$ (blue triangle) and the black line is the Nasmyth spectrum for the estimated rate of dissipation. files were successfully transmitted to the glider's shore-side command computer after each dive.

The rationale for making multiple deployments was to test different shear probe orientations and probe angles, primarily to investigate the effect on low-wavenumber suppression of shear, as observed in the Puget Sound. Increasing the angle of the MicroPods from $0^{\circ}$ (parallel to the major axis of the glider) increases the distance between the probes and the body of the glider and changes the angle of the probes to the direction of travel. The four probe configurations tested were: (a) shear probe orientation - vertical, probe angle - $0^{\circ}(48 \mathrm{~mm}$ from glider body); (b) shear probe orientation - vertical, probe angle - $4^{\circ}(64 \mathrm{~mm})$; (c) shear probe orientation - vertical, probe angle - $8^{\circ}(78 \mathrm{~mm})$; (d) shear probe orientation - lateral, probe angle $0^{\circ}(48 \mathrm{~mm})$.

Configuration A was identical to that used in the Puget Sound and the low-wavenumber suppression of shear that was seen in the Puget Sound data was also observed in the Loch Linnhe data. Increasing the probe angle (configurations B and C) did not make a noticeable difference to the extent of the shear suppression. However, orientating the shear probe laterally (i.e. measuring $\partial \mathrm{v} / \partial \mathrm{x}$; configuration D) successfully reduced the low-wavenumber shear suppression such that the shear spectra closely follow the Nasmyth spectrum, Fig. 6. Trials with lateral shear probe orientation and probe angle $>0^{\circ}$ will be undertaken in the future, as well as detailed comparison of the downward and upward profiles.

The microstructure data was successfully integrated with the Seaglider data stream during post-processing. The highresolution temperature profiles from the FP07 thermistor closely match the low-resolution profiles from the glider CTD after a uniform offset of $0.68^{\circ} \mathrm{C}$ was applied, Fig. 7a. The CTD data were used to calculate profiles of buoyancy

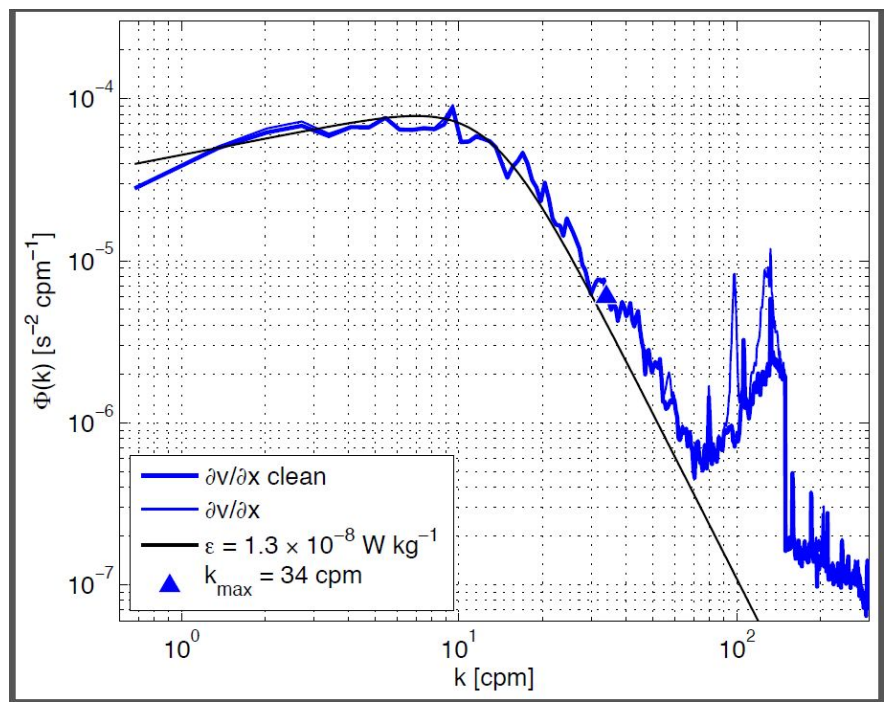

Fig. 6. Spectrum of lateral shear $(\partial v / \partial x$, thin blue line) from Loch Linnhe in the pressure range of 105 to $130 \mathrm{dbar}$. The thick blue line is the spectrum of shear after removing contributions that are coherent with the lateral vibrations sensed by the piezo-accelerometer. The limit of spectral integration is $34 \mathrm{cpm}$ (blue triangle) and the black line is the Nasmyth spectrum for the estimated rate of dissipation. The probe angle was $0^{\circ}$ and the profiling speed was $0.37 \mathrm{~m} \mathrm{~s}^{-1}$. 
frequency squared, $\mathrm{N}^{2}=-\mathrm{g} / \rho \partial \rho / \partial \mathrm{z}$, where $\rho$ is potential density (a function of temperature, salinity and pressure), Fig. $7 \mathrm{~b}$. Profiles of turbulent kinetic energy dissipation rate, $\varepsilon$, were then calculated from (1) in 8-second, 50\%-overlapping bins, and combined with the profiles of buoyancy frequency squared to yield estimates of vertical eddy diffusivity, $\mathrm{K}_{\mathrm{z}}=0.2 \varepsilon / \mathrm{N}^{2}[1]$, Fig. 7d.

Patches of elevated turbulence are observed near the surface and at mid depth (105 to 130 dbar). The latter is associated with a temperature inversion, compensated for by an increase in salinity so that potential density continues to increase with depth. Both shear, Fig. 7c and temperature gradient (not shown) variance are high in this patch and the former is used to estimate turbulent kinetic energy dissipation rates on the order of $10^{-8} \mathrm{~W} \mathrm{~kg}^{-1}$ and vertical eddy diffusivities in the range $10^{-4}$ to $3 \times 10^{-3} \mathrm{~m}^{-2} \mathrm{~s}^{-1}$. Turbulent mixing rates of this magnitude are typical in tidally energetic sea lochs.

\section{CONCLUSION}

A microstructure sensor suite consisting of two MicroPod probe modules was integrated into a Seaglider and performed well in a series of laboratory and sea trials. Shear probes that were mounted in an orientation to measure the vertical shear component, i.e. normal to the hull of the glider, showed evidence of low-wavenumber shear signal suppression, which is caused by the proximity of probe to the hull. Orientation of the shear probe to measure the lateral shear component, i.e. tangential to the glider hull, did not show evidence of such low-wavenumber signal suppression. Further testing of the effect of shear probe orientation and probe angle on lowwavenumber shear suppression is planned, as well as the

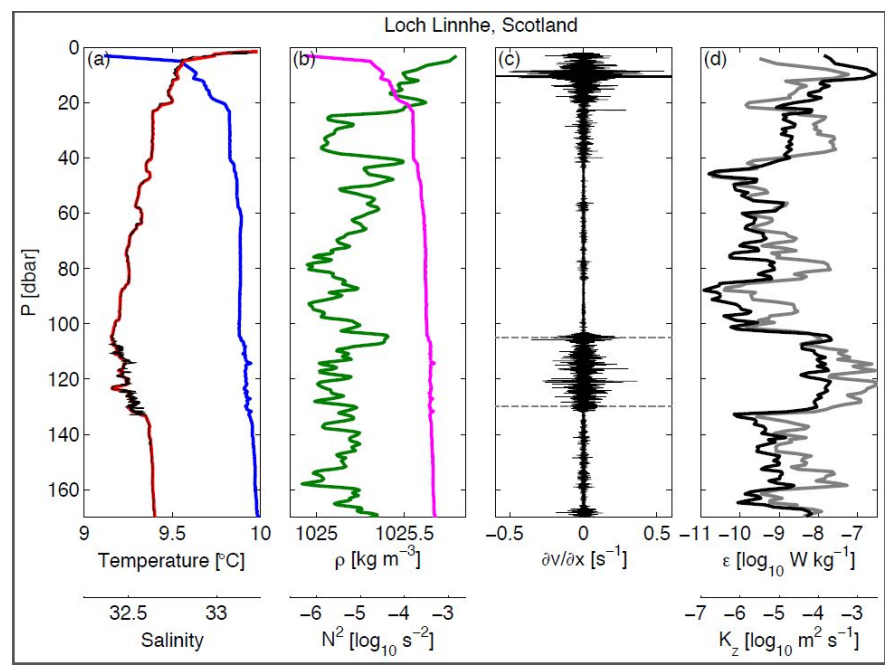

Fig. 7. Example data from Loch Linnhe: (a) Profiles of temperature (red line) and salinity (blue) measured by the glider CTD along with a high-resolution temperature profile from the FP07 thermistor (thin black line). (b) Profiles of potential density ( $\rho$, magenta) and buoyancy frequency squared $\left(\mathrm{N}^{2}\right.$, green). (c) Profile of lateral shear band-passed filtered between 0.15 and $15 \mathrm{~Hz}$. The dashed lines indicate the pressure window of the shear spectrum shown in Figure 6. (d) Profiles of turbulent kinetic energy dissipation rate ( $\varepsilon$, black line) and vertical eddy diffusivity $\left(\mathrm{K}_{\mathrm{z}}\right.$, grey line). development of routines to further constrain estimates of glider speed and angle of attack.

The presented engineering work and field tests demonstrate the successful integration of the turbulence sensors system into the Seaglider. The availability of turbulence sensors on the Seaglider platform provides researchers with the opportunity to conduct long-term, contiguous turbulence measurements, which will provide data need to understand the temporal and spatial variability of the turbulent processes in the oceans.

\section{ACKNOWLEDGMENTS}

The authors would like to thank: Dave Cronkrite and Rick Noel for their help in building the RSI MicroPods and the DataLogger electronics; Ross Healy and David Nichols for their assistance in designing, assembling and modifying the Seaglider MicroPod mount as well as assisting during the Puget Sound sea trials; and Bastien Queste and Ryan Gilchrist for assisting and piloting during the Loch Linnhe sea trial.

\section{REFERENCES}

[1] T.R. Osborn, "Estimates of the local rate of vertical diffusion from dissipation measurements," J. Phys. Oceanogr., vol. 10 , pp. 83-89. 